Document downloaded from:

http://hdl.handle.net/10251/83822

This paper must be cited as:

Budzko, D.; Hueso Pagoaga, JL.; Martínez Molada, E.; Teruel-Ferragud, C. (2016). Dynamical study while searching equilibrium solutions in N-body problem. Journal of Computational and Applied Mathematics. 297:26-40. doi:10.1016/j.cam.2015.11.010.

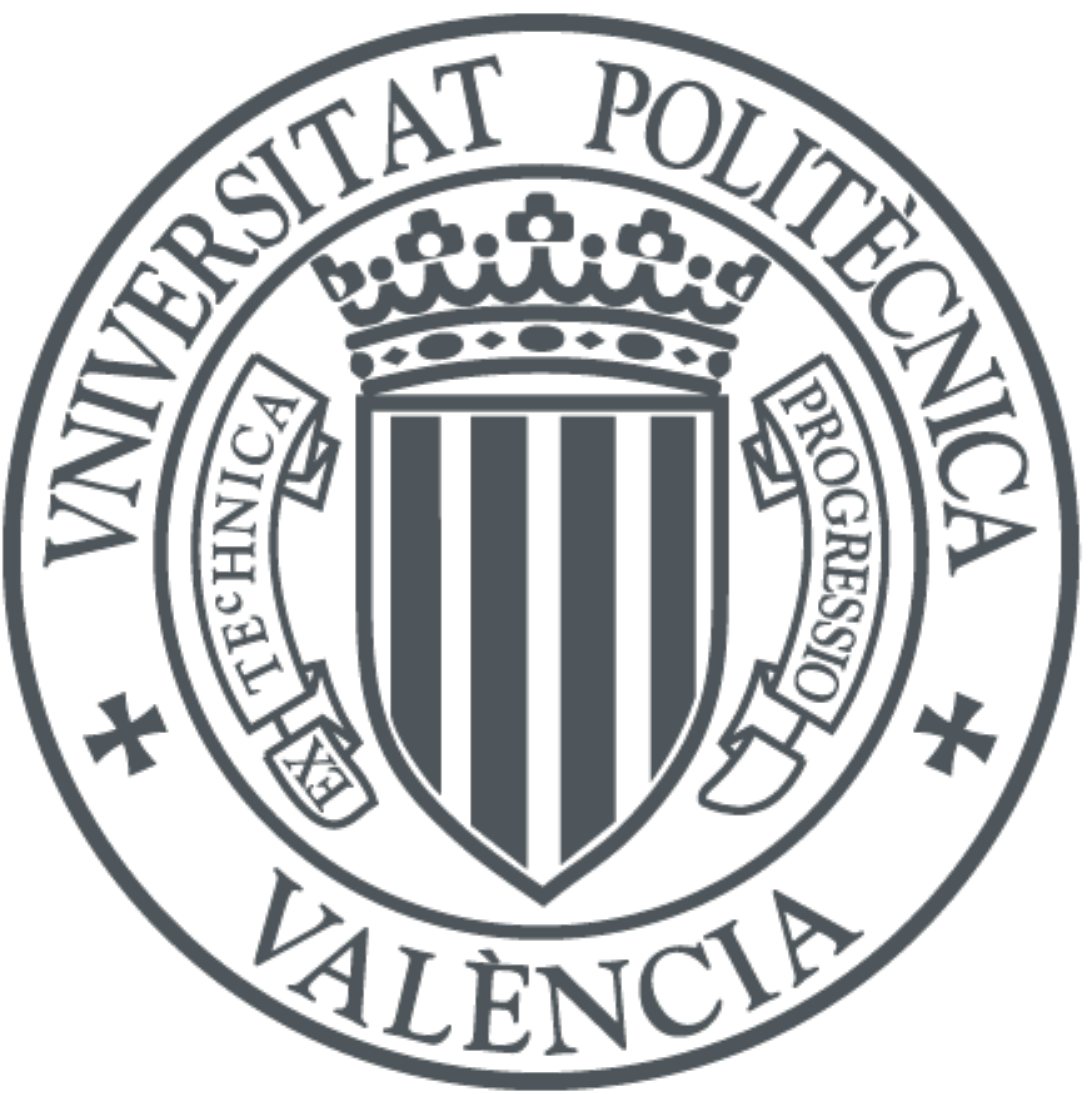

The final publication is available at

http://dx.doi.org/10.1016/j.cam.2015.11.010

Copyright Elsevier

Additional Information 


\title{
Dynamical Study while Searching Equilibrium Solutions in $N$-body Problem ${ }^{\text {th }}$
}

\author{
Dzmitry A. Budzko ${ }^{\text {, José L. Hueso }}{ }^{\text {b,* }}$, Eulalia Martínez ${ }^{\mathrm{b}}$, Carles Teruel ${ }^{\mathrm{b}}$ \\ ${ }^{a}$ University of Brest, Belarus \\ ${ }^{b}$ Universitat Politècnica de València, Spain
}

\begin{abstract}
The theory of complex dynamics is usually applied to compare the global convergence properties of different iterative methods, by obtaining the attraction basins for simple polynomial equations in the complex domain. However, in this work, we use it in quite another context: the study of a nontrivial nonlinear system that describes the motion of interacting bodies in celestial mechanics, namely, Newtonian planar circular restricted four-body problem and its relative equilibrium solutions. These have been investigated from a dynamical point of view. New properties of the solutions of this system have been obtained. Practical guidelines for efficient search of relative equilibrium solutions of $N$-body problem have been given.
\end{abstract}

Keywords: N-body problem, Equilibrium points, Complex dynamics, Nonlinear systems, Newton's method, Series approximations

\section{Introduction}

The dynamics of different iterative methods for the solution of nonlinear equations has been widely studied (see, for example [1]-[3] and the references therein) by analysing the properties of the rational functions in the complex plane that arise when applying the method to polynomials of certain degree.

The dynamics in the real plane has been analyzed mainly for birational maps [4] or quadratic maps [5]. The study in the real plane is more involved because the powerful tools of complex analysis such as Montel's theorem [6] for holomorphic functions cannot be applied here.

Our objective is to study the dynamics of Newton's method applied to a nonlinear system in $\mathbb{R}^{2}$ that is of interest in celestial mechanics. Our results show that the behaviour of the discrete dynamical system here considered resembles more the dynamics of rational functions in the complex plane than that of the birational or quadratic maps in the real plane.

\footnotetext{
This research has been supported by Ministerio de Ciencia y Tecnología MTM2014-52016-C2-02

* Corresponding author

Email addresses: dzmitry.budzko@gmail.com (Dzmitry A. Budzko), jlhueso@mat.upv.es (José L. Hueso ), eumarti@mat.upv.es (Eulalia Martínez ), cartefer@teleco.upv.es (Carles Teruel)
} 
In the classical $N$-body problem, the search of relative equilibrium solutions is a very intricate problem itself, because the number of real solutions increases very quickly while the number of interacting bodies $N$ grows and at present nobody knows even how to find the number of all equilibrium solutions (or central configurations) for arbitrary $N$. Only few such results are known for small $N(N=2,3,4)$ or for some particular, for example, symmetric cases. The equations that determine relative equilibrium solutions in the $N$-body problem are nonlinear algebraic equations and they usually contain some geometric or dynamic parameters.

In this paper we consider the equilibrium solutions in the Newtonian planar circular restricted four-body problem [7]-[9], formulated on the basis of Lagrange's triangular solutions. The system that determines the equilibrium positions of the body of infinitesimal mass,

$$
\begin{aligned}
(\sqrt{3} x-y)\left(1-\frac{1}{\left(x^{2}+y^{2}\right)^{3 / 2}}\right)+\mu_{1}(\sqrt{3}(x-1)+y)\left(1-\frac{1}{\left((x-1)^{2}+y^{2}\right)^{3 / 2}}\right) & =0 \\
2 y\left(1-\frac{1}{\left(x^{2}+y^{2}\right)^{3 / 2}}\right)+\mu_{2}(\sqrt{3}(x-1)+y)\left(1-\frac{1}{\left((x-1 / 2)^{2}+(y-\sqrt{3} / 2)^{2}\right)^{3 / 2}}\right) & =0,
\end{aligned}
$$

has from 8 to 10 solutions, depending on two mass parameters $\mu_{1}$ and $\mu_{2}$. Parameters $\mu_{1}$ and $\mu_{2}$ are mass ratios and are chosen in such a way to be varied from zero to one. This system is of great interest because its study facilitates the construction of motion theory of asteroids near Lagrange's triangular libration points. In the solar system the most known such configuration is formed by the Sun, Jupiter and Trojan asteroids.

Each equation defines a curve in the plane XY and the solutions are located at the points of intersection of these two curves. On Figure 1 these curves are drawn in bold solid and dashed lines, respectively, for the values of the parameters $\mu_{1}=0.9$ and $\mu_{2}=$ 0.05 . The bigger points represent the canonical position of the three massive bodies, denoted by $P_{0}, P_{1}$ and $P_{2}$. The solutions are the remaining intersections, which are labelled as $S_{1}$ to $S_{8}$. Points $L_{1}$ to $L_{5}$ are the equilibrium solutions of the corresponding restricted three body problem, known as Lagrange's libration points.

In order to determine the equilibrium points, we apply Newton's method [10] to this system, starting from a given initial approximation. Newton's iterations generally converge to a solution, but it is not easy to predict which one, as it depends on the starting point. The attraction basin of a root is formed by the starting points which Newton's iterations converge to this root. We find that the attraction basins of the roots of this system are very irregular, chaotic and full of noise, especially if parameters $\mu_{1}$ and $\mu_{2}$ are close to zero (the most interesting case for applications). This means that the problem of choosing initial estimations for the iterative method is very sensitive and needs careful consideration, because the next step is the stability analysis of every solution under any values of parameters. We have confronted the numerical solutions with the solutions obtained in the form of power series [11] in order to determine if the series approximations are suitable as starting points for the Newton's iterations.

The rest of the paper is organized as follows. In section 2 we analyse the behaviour of the iterations of Newton's method for the solution of system (1) for different values of the parameters $\mu_{1}$ and $\mu_{2}$ by obtaining the basins of attraction. Section 3 is devoted 


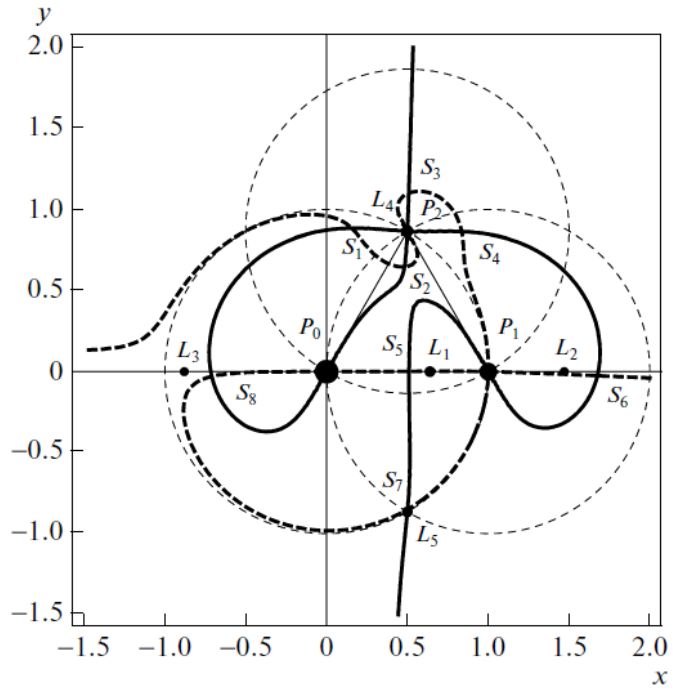

Figure 1: Solutions of the four-body problem for $\mu_{1}=0.9$ and $\mu_{2}=0.05$.

to the construction of series approximations to the solutions. In section 4 we assess the suitability of such approximations as starting points for the Newton's iterations to converge to the corresponding solution and the last section is devoted to the conclusions.

\section{Attraction basins for Newton's method}

Let us recall some concept on dynamics. If $G: \mathbb{R}^{n} \longrightarrow \mathbb{R}^{n}$ is Fréchet differentiable, given $x \in \mathbb{R}^{n}$, then the orbit of $x$ is the set

$$
\left\{x, G(x), G^{2}(x), \ldots, G^{p}(x), \ldots\right\} .
$$

A point $x_{f} \in \mathbb{R}^{n}$ is a fixed point of $G$ if $G\left(x_{f}\right)=x_{f}$. Let $D G(x)$ the Jacobian of F. If $\left\|D G\left(x_{f}\right)\right\|<1$ the fixed point is attracting and if $D G\left(x_{f}\right)=0$ the fixed point is superattracting.

Let $x_{f}$ be an attracting fixed point of $\mathrm{G}$. The attraction basin of $x_{f}$ is the set of points whose orbits tend to that fixed point.

Consider a system of nonlinear equations $F(x)=0$, where $F: \mathbb{R}^{n} \longrightarrow \mathbb{R}^{n}$ is a Fréchet differentiable function. The application of Newton's method to the solution of the system can be interpreted as a discrete dynamical system $x_{k+1}=G\left(x_{k}\right)$ where $G$ is the operator in $\mathbb{R}^{n}$ given by

$$
G(x)=x-D F(x)^{-1} F(x)
$$




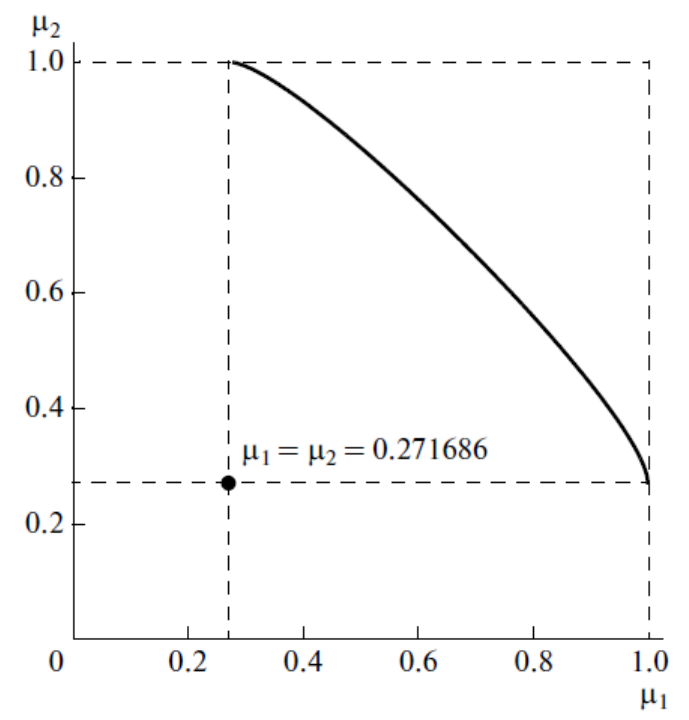

Figure 2: Bifurcation curve in the plane $\left(\mu_{1}, \mu_{2}\right)$.

Under the conditions of Kantorovich's theorem, the zeros of $F$ are superattracting points of $G$.

Here we consider the iterative function $G$ obtained by applying Newton's method to system (1) for given values of the parameters $\mu_{1}$ and $\mu_{2}$ and explore some of the properties of the attraction basins of the solutions.

In order to find the attraction basins, we fix a pair of values to the parameters, take the points of a mesh in a rectangle in the plane XY as initial estimations and iterate Newton's method until the tolerance is satisfied or the maximum number of iterations is reached. Assigning a colour to each solution of system (1), we give that colour to all the starting points whose Newton's iterations converge to the corresponding solution. In this way we visualize the attraction basins of the roots, which have been previously obtained numerically, by applying the algorithms proposed in [11].

In [7] the authors analyse the number of solutions of the system for different values of parameters $\mu_{1}$ and $\mu_{2}$ finding a bifurcation curve that divides the rectangle $\left(\mu_{1}, \mu_{2}\right) \in$ $[0,1] \times[0,1]$ in two zones, as seen on Figure 2. For parameter values below the curve, the system presents 8 solutions, 10 above the curve in the upper part and 9 along it. It is interesting to see how the basins change with the number of solutions and what happens near the bifurcation curve.

Fixing the value of $\mu_{1}=0.9$ and giving different values to $\mu_{2}$, we examine different cases for the solutions. The bifurcation curve appears about $\mu_{2}=0.44$. For lower values of $\mu_{2}$ there are 8 solutions and for higher values 10. In Figures 3 and 4 we compare the basins of attraction of Newton's method for $\mu_{2}=0.438$ and $\mu_{2}=0.442$, 


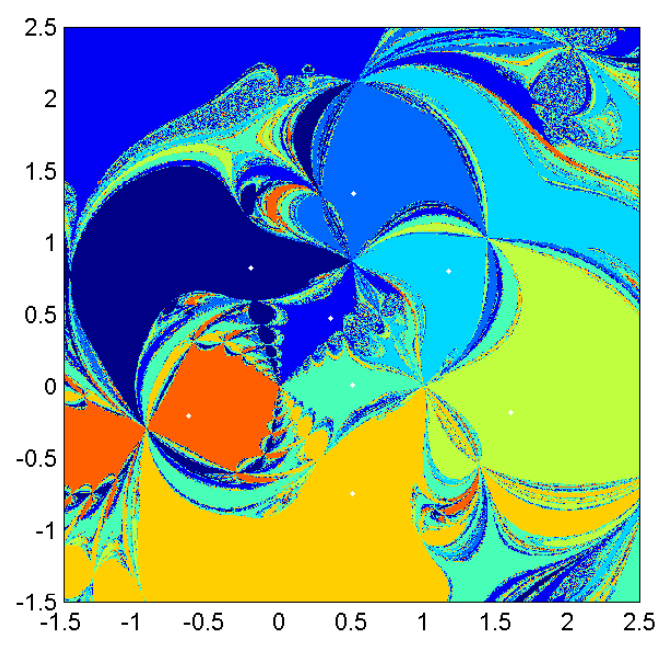

Figure 3: Below the bifurcation curve, for $\mu_{1}=0.9, \mu_{2}=0.438$, there are 8 basins of attraction.

one below the bifurcation curve and the other above it. In the first case there are 8 basins. In the centre of the image, the basins are quite intricate, indicating the proximity of the bifurcation. The second case, for $\mu_{2}=0.442$, presents 10 attraction basins. The fuzzy zones in the former figure are mainly occupied by basins corresponding to the two new equilibrium points.

When the parameter values are very different, the basins become asymmetric as shown in Figure 5 corresponding to the parameter values $\mu_{1}=0.9$ and $\mu_{2}=0.0001$.

For small values of the parameters, the basins are very intricate. The bigger the parameters, the simpler the basins, but they always have a fractal appearance. If $\mu_{1}=$ $\mu_{2}$, then the basins present a symmetry of axis $y=x / \sqrt{3}$. Figures 6 and 7 compare the attraction basins for big and small values of the parameters.

The basins geometry indicates the difficulty to choose a suitable starting point that guarantees the convergence of the iterative method to the desired solution. Figures 8 shows that for some solutions there are points very close to them belonging to the attraction basin of another solutions.

Nevertheless, Newton's method almost always converges to a solution, independently of the parameter values and the starting point, which is quite remarkable given the complexity of the system of equations.

The following result gives information about the boundedness of the attracion bassins.

Theorem 1. The iteration function $G$ of Newton's method applied to system (1) has at most one unbounded basin.

Proof: Let $\left(x_{0}, y_{0}\right)$ a point far enough from the origin and $\left(x_{1}, y_{1}\right)$ the result of a New- 


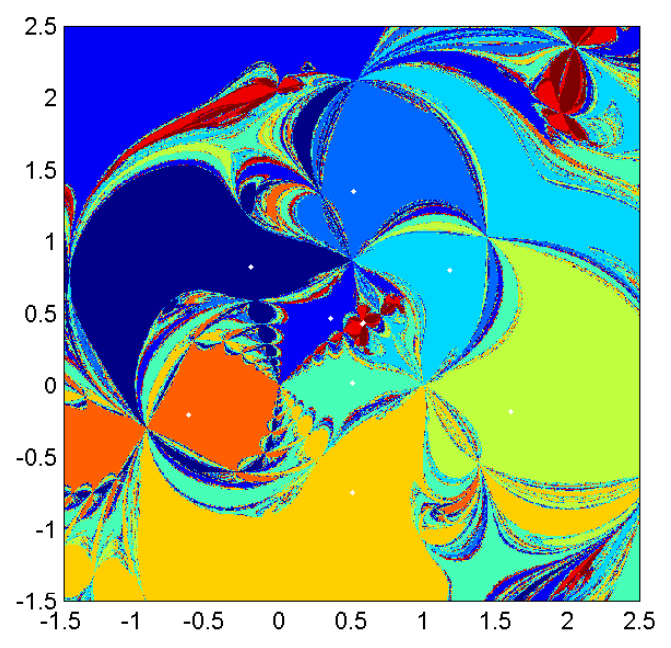

Figure 4: Above the bifurcation curve, for $\mu_{1}=0.9, \mu_{2}=0.442$, two new basins appear.

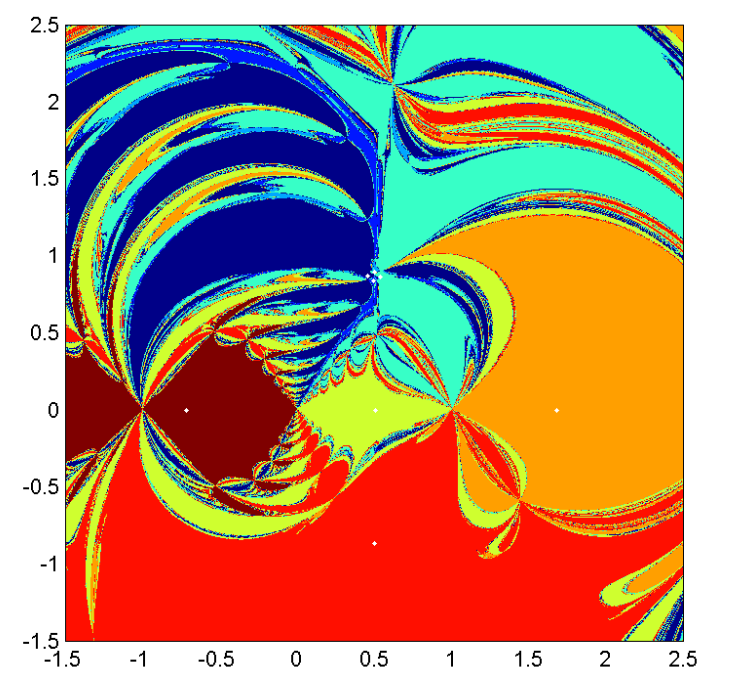

Figure 5: Basins asymmetry for $\mu_{1}=0.9, \mu_{2}=0.0001$. 


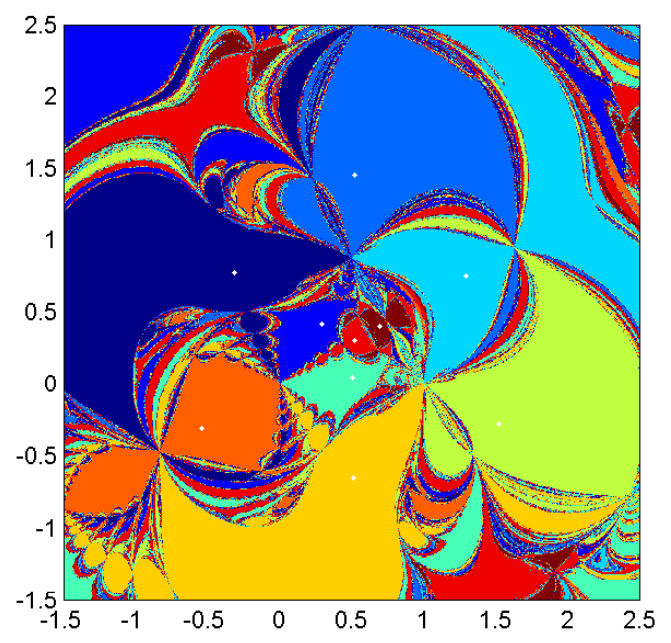

Figure 6: Attraction basins for $\mu_{1}=\mu_{2}=0.9$.

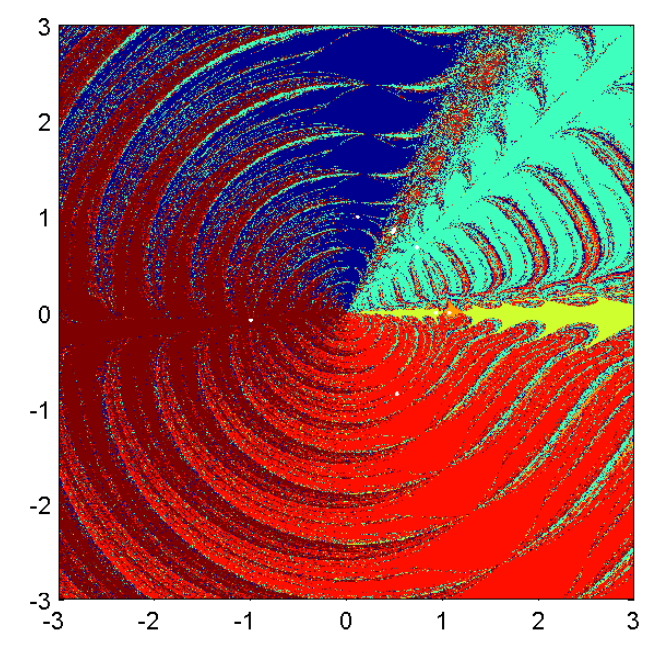

Figure 7: Attraction basins for $\mu_{1}=10^{-3}$ and $\mu_{2}=10^{-4}$. 


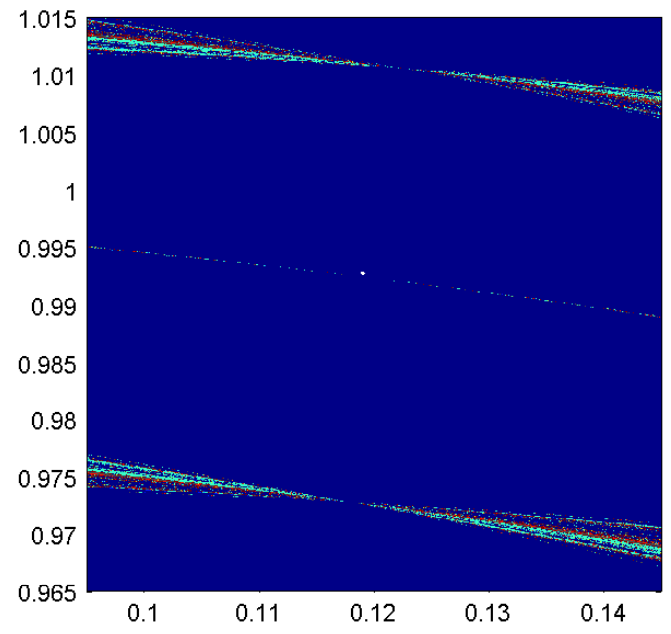

Figure 8: Detail of the attraction basins of solution $S_{1}$ for $\mu_{1}=10^{-3}$ and $\mu_{2}=10^{-4}$ showing nearby points belonging to other basins.

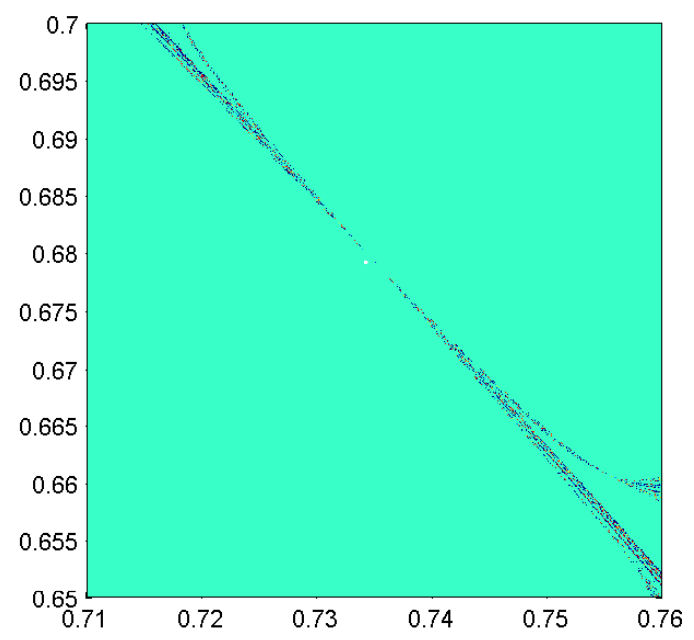

Figure 9: Detail of the attraction basin of solutions $S_{4}$ for $\mu_{1}=10^{-3}$ and $\mu_{2}=10^{-4}$. 
ton's iteration on system (1). If we consider the linear system

$$
\begin{array}{r}
(\sqrt{3} x-y)+\mu_{1}(\sqrt{3}(x-1)+y)=0 \\
2 y+\mu_{2}(\sqrt{3}(x-1)+y)=0,
\end{array}
$$

one step of Newton's method, independently from the starting point, gives the solution

$$
\begin{aligned}
& \bar{x}=\frac{\mu_{1}+\mu_{2} / 2}{1+\mu_{1}+\mu_{2}} \\
& \bar{y}=\frac{\sqrt{3} \mu_{2} / 2}{1+\mu_{1}+\mu_{2}} .
\end{aligned}
$$

The iterate $\left(x_{1}, y_{1}\right)$ is very close to $(\bar{x}, \bar{y})$ because, for $\left(x_{0}, y_{0}\right)$ big enough, both $F\left(x_{0}, y_{0}\right)$ and $D F\left(x_{0}, y_{0}\right)$ are very close to the corresponding values in system (2). Thus, all the points far enough from the origin will be in the same attraction basin as $(\bar{x}, \bar{y})$, so that, the other basins will be bounded.

Observe that $(\bar{x}, \bar{y})$ is the centre of mass of the physical system of the three bodies. For not too small values of $\mu_{1}$ and $\mu_{1}$, the centre of mass is in the attraction basin of the solution closer to it. For example, for $\mu_{1}=0.1, \mu_{2}=0.01$, taking starting points far enough from the origin, Newton's iterations converge to $S_{5}(0.7176,0.0006)$ that is the solution closer to the centre of mass. However, for $\mu_{1}=\mu_{2}=0.001$ the unbounded basin corresponds to the root $S_{4}(0.8665,0.5003)$ whose distance to the centre of mass is 1 , but there are two different solutions closer to it, namely $S_{2}(0.4662,0.8074)$ and $S_{5}(0.9323,0.0)$ at distance 0.93 .

Figures 10 and 11 show a wide view of the attraction basins for the considered parameter values.

\section{Power series solutions}

This section is devoted to the construction of a solution in the form of a power series. If we are interested mostly in case $\mu_{1}>\mu_{2}$ then it is expedient to expand the power series in terms of a small parameter $\mu_{2}$. In this way the expansions will be more accurate. But if it is required to consider the case $\mu_{1}<\mu_{2}$ then the accuracy would be better if the power series expansion were carried out in terms of small parameter $\mu_{1}$.

So, for small values of $\mu_{2}$, the roots $S(x, y)$ of system (1) can be approximated [12] by the partial sums $s_{n}=\left(u_{n}, v_{n}\right)$ of a power series

$$
\begin{aligned}
& x \simeq u_{n}=\sum_{j=0}^{n} x_{j} \mu_{2}^{j k}=x_{0}+x_{1} \mu_{2}^{k}+x_{2} \mu_{2}^{2 k}+x_{3} \mu_{2}^{3 k}+\ldots+x_{n} \mu_{2}^{n k}, \\
& y \simeq v_{n}=\sum_{j=0}^{n} y_{j} \mu_{2}^{j k}=y_{0}+y_{1} \mu_{2}^{k}+y_{2} \mu_{2}^{2 k}+y_{3} \mu_{2}^{3 k}+\ldots+y_{n} \mu_{2}^{n k}
\end{aligned}
$$

where the zero approximation of the $\operatorname{root}\left(x_{0}, y_{0}\right)$ is determined by the condition $\mu_{2}=0$. These expansions theoretically should give better estimations while $n$ grows, assuming 


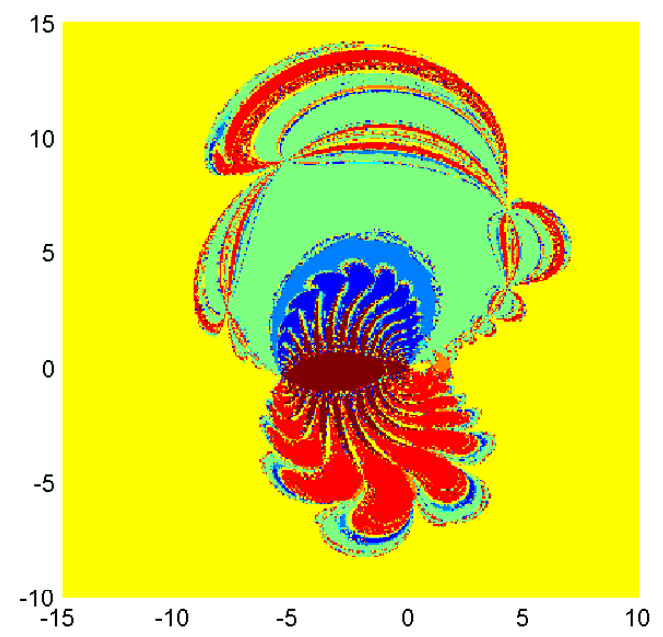

Figure 10: Attraction basins for $\mu_{1}=0.1$ and $\mu_{2}=0.01$.

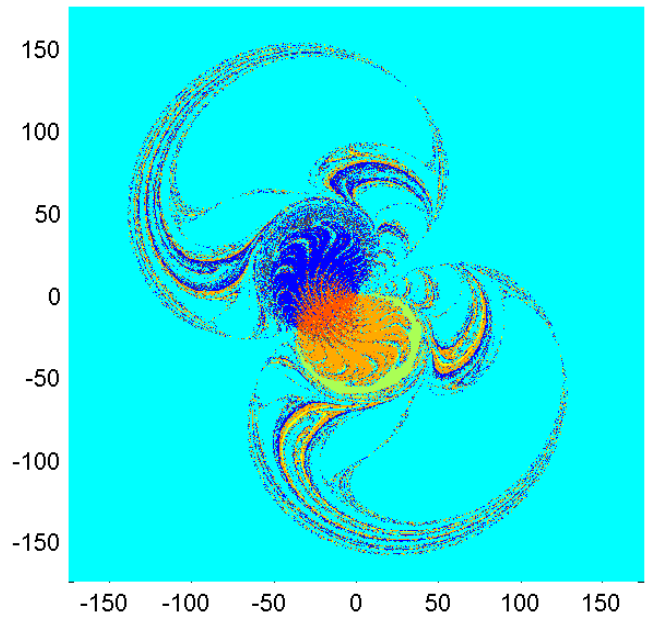

Figure 11: Attraction basins for $\mu_{1}=\mu_{2}=0.001$ 
that the corresponding series converge. As it was shown [13] in 1912, the series for the restricted three-body problem is convergent, but the rate of convergence is extremely slow.

When $\mu_{2}=m_{2} / m_{0}=0$ then it means that the masses of two bodies are equal to zero, and there are only two bodies with nonzero masses. Therefore the considered restricted four-body problem is reduced to the famous restricted three-body problem [14], which has five equilibrium solutions known as libration points. It means that there are exactly five zero approximations $\left(x_{0}, y_{0}\right)$ for the expansions (6). The coordinates of three collinear libration points of Euler's type, $L_{1}, L_{2}$ and $L_{3}$ are dependent on $\mu_{1}$. The two triangular libration point of Lagrange's type, $L_{4}(1 / 2, \sqrt{3} / 2)$ and $L_{5}(1 / 2,-\sqrt{3} / 2)$ do not depend on $\mu_{1}$. In this paper, for convenience, we take only Lagrange's triangular libration points as zero approximations in (6).

Following [11], in order to find the coefficients $\left(x_{j}, y_{j}\right), j=1,2, \ldots$ of the expansion and determine the value of exponent $k$, it is required to substitute expressions (6) into (1), to expand each equation into a power series in terms of $\mu_{2}$, and to equate the coefficients of the terms with identical powers of the left and right sides of each equation. Finally, we obtain a system of equations in the desired coefficients $\left(x_{j}, y_{j}\right)$. Note that in the neighborhood of point $x_{0}=1 / 2, y_{0}=\sqrt{3} / 2$, a solution of system (1) in form (6) exists only for $k=1 / 3$ and it is required to immediately substitute $(1 / 2, \sqrt{3} / 2)$ for the zero approximation $\left(x_{0}, y_{0}\right)$ into formula (6), since the coefficients of $\mu_{2}$ in (1) turn to infinity at this point. The two first equations of this obtained system contain only $x_{1}$ and $y_{1}$ and have the form

$$
\begin{aligned}
& x_{1}\left(-3 \mu_{1}+\frac{4}{\left(x_{1}^{2}+y_{1}^{2}\right)^{3 / 2}}-3\right)+3 \sqrt{3}\left(\mu_{1}-1\right) y_{1}=0 \\
& 3 \sqrt{3}\left(\mu_{1}-1\right) x_{1}+y_{1}\left(-9 \mu_{1}+\frac{4}{\left(x_{1}^{2}+y_{1}^{2}\right)^{3 / 2}}-9\right)=0 .
\end{aligned}
$$

One can find out that equations (7) have four real solutions and it is possible to write them in explicit form. The third and fourth equations of the system that determines coefficients $\left(x_{j}, y_{j}\right)$ depend only on $x_{1}, x_{2}, y_{1}, y_{2}$ and have the following form:

$$
\begin{array}{r}
x_{1}^{2}\left(21\left(\mu_{1}-1\right)-\frac{32 x_{2}}{\left(x_{1}^{2}+y_{1}^{2}\right)^{5 / 2}}\right)+6 x_{1} y_{1}\left(\sqrt{3}\left(\mu_{1}+1\right)-\frac{8 y_{2}}{\left(x_{1}^{2}+y_{1}^{2}\right)^{5 / 2}}\right)+ \\
+4 x_{2}\left(-3 \mu_{1}+\frac{4 y_{1}^{2}}{\left(x_{1}^{2}+y_{1}^{2}\right)^{5 / 2}}-3\right)-3\left(\mu_{1}-1\right)\left(11 y_{1}^{2}-4 \sqrt{3} y_{2}\right)=0 \\
12 \sqrt{3}\left(\mu_{1}-1\right) x_{2}+x_{1}^{2}\left(3 \sqrt{3}\left(\mu_{1}+1\right)+\frac{16 y_{2}}{\left(x_{1}^{2}+y_{1}^{2}\right)^{5 / 2}}\right)+ \\
+6 x_{1} y_{1}\left(-11 \mu_{1}-\frac{8 x_{2}}{\left(x_{1}^{2}+y_{1}^{2}\right)^{5 / 2}}+11\right)- \\
-\frac{32 y_{1}^{2} y_{2}}{\left(x_{1}^{2}+y_{1}^{2}\right)^{5 / 2}}+9 \sqrt{3} \mu_{1} y_{1}^{2}-36 \mu_{1} y_{2}+9 \sqrt{3} y_{1}^{2}-36 y_{2}=0 .
\end{array}
$$


The system (8) is linear in $x_{2}, y_{2}$, so after substituting the corresponding solution of (7) into (8), this system will still have four solutions. The corresponding fifth and sixth equations contain only $x_{1}, x_{2}, x_{3}, y_{1}, y_{2}, y_{3}$ and they are quite cumbersome, so we do not write these equations here. But they are also linear in $x_{3}, y_{3}$ and so, the general system for determining $x_{j}, y_{j}$ remains to still have four solutions.

We denote by $s_{j}^{i}=\left(u_{j}^{i}, v_{j}^{i}\right)$ the point whose coordinates are the $j$-th partial sums (6) corresponding to the $i$-th set of coefficients, $i=1, \ldots 4$.

For example, for $\mu_{1}=0.3\left(x_{0}=1 / 2, y_{0}=\sqrt{3} / 2, k=1 / 3\right)$ the corresponding coefficients in (6) can be written as a set of four subsets

$$
\begin{gathered}
\left\{x_{1}=-1.09303, y_{1}=0.430598, x_{2}=-0.601083,\right. \\
\left.y_{2}=-0.176974, x_{3}=-0.520212, y_{3}=-0.81574\right\}, \\
\left\{x_{1}=0.246611, y_{1}=0.625996, x_{2}=-0.0418395,\right. \\
\left.y_{2}=0.140344, x_{3}=0.029317, y_{3}=-0.0633965\right\}, \\
\left\{x_{1}=-0.246611, y_{1}=-0.625996, x_{2}=-0.0418395,\right. \\
\left.y_{2}=0.140344, x_{3}=-0.029317, y_{3}=0.0633965\right\}, \\
\left\{x_{1}=1.09303, y_{1}=-0.430598, x_{2}=-0.601083,\right. \\
\left.y_{2}=-0.176974, x_{3}=0.520212, y_{3}=0.81574\right\}
\end{gathered}
$$

Expanding around the second zero approximation $x_{0}=1 / 2, y_{0}=-\sqrt{3} / 2$ we obtain a unique solution of the form

$$
\begin{array}{r}
x \simeq u_{3}=-\frac{\left(\mu_{1}-1\right)\left((659 \sqrt{3}-783) \mu_{1}^{2}+(3942-152 \sqrt{3}) \mu_{1}+659 \sqrt{3}-783\right) \mu_{2}^{3}}{59049 \mu_{1}^{3}}- \\
-\frac{(12 \sqrt{3}-95)\left(\mu_{1}^{2}-1\right) \mu_{2}^{2}}{729 \mu_{1}^{2}}+\frac{(\sqrt{3}-9)\left(\mu_{1}-1\right) \mu_{2}}{27 \mu_{1}}+\frac{1}{2}, \\
y \simeq v_{3}=-\frac{\sqrt{3}}{2}+\frac{(3 \sqrt{3}-1)\left(\mu_{1}+1\right) \mu_{2}}{27 \mu_{1}}+ \\
+\frac{\left((17 \sqrt{3}-36) \mu_{1}^{2}+(90-218 \sqrt{3}) \mu_{1}+17 \sqrt{3}-36\right) \mu_{2}^{2}}{2187 \mu_{1}^{2}}- \\
-\frac{\left(\mu_{1}+1\right)\left((1917 \sqrt{3}-1627) \mu_{1}^{2}+(3592-5238 \sqrt{3}) \mu_{1}+1917 \sqrt{3}-1627\right) \mu_{2}^{3}}{59049 \mu_{1}^{3}} .
\end{array}
$$

Note that the value of exponent $k$ is equal to unity for this starting point. The approximations $\left(u_{j}, v_{j}\right)$ generated by $(10)$ are denoted by $s_{j}^{-}$.

\section{Initial estimations}

The objective of this section is to assess the suitability of the series approximations of the solutions as starting points for the Newton's iterations to converge to the desired solution.

We consider a discrete mesh of pairs $\left(\mu_{1}, \mu_{2}\right)$ in the rectangle $[0,1] \times[0,1]$. For each point in the mesh, we compute the Newton's iterations starting from a partial sum 


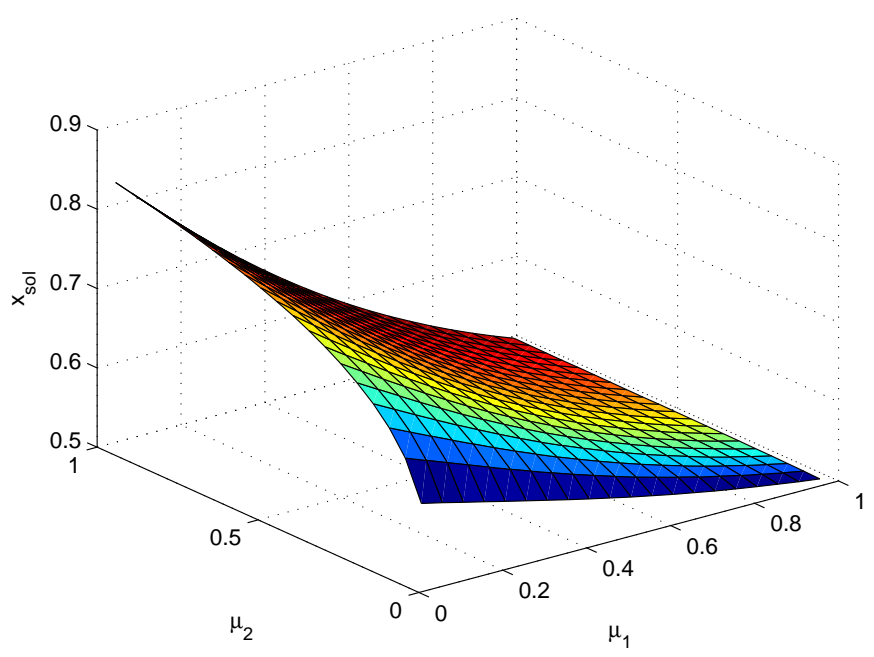

Figure 12: The series approximations $s_{j}^{3}$ of any order $j$ are $S_{3}$-suitable for all $\left(\mu_{1}, \mu_{2}\right)$.

of the series considered in the former section and see if they converge and to which solution. The pairs $\left(\mu_{1}, \mu_{2}\right)$ for which $s_{j}^{i}$ belongs to the attraction basin of $S_{k}$ constitute the $S_{k}$-suitability region of the $j$-th partial sum of the series $i$.

The results can be displayed by using surfaces in three dimensions. The first two dimensions correspond to the parameter values. The height and the colour of a point are the abscissa and the ordinate of the last Newton's iteration. As the equilibrium points vary continuously with the parameters, sharp changes in the surface height or colour indicate the parameter values for which the iterations converge to the different solutions. Oppositely, a smooth surface shows that the iterations converge to a given (variable) solution for all the parameter values. To ease the interpretation, the surface is projected on the $\left(\mu_{1}, \mu_{2}\right)$-plane so that the information can be related to the parameter values.

Let us first examine the series approximations to the equilibrium points $S_{1}, S_{2}, S_{3}$ and $S_{4}$ generated by formula (6). The approximations of $s_{j}^{3}$ for $j=0,1, \ldots$ and for any values of the parameters $\mu_{1}$ and $\mu_{2}$ are $S_{3}$ suitable in the sense above defined, as it can be seen in Figure 12. This figure is shown in three dimensions, where the height represents the abscissa of the last iteration and the colour its ordinate.

The other equilibrium points do not present such a simple behaviour. For the equilibrium point $S_{1}$, the partial sums $s_{j}^{1}$ provide suitable estimations for $\mu_{1}>0.25$. For $\mu_{1}<0.2$, the suitability region depends on the order of the sum, as shown in Figures 13 and 14. Experimentally we find that the estimations are suitable for $\mu_{2} \leq 20 \mu_{1}^{2}$. Figures 15 and 16 show the suitability regions near the origin.

On the contrary, for $S_{2}$, small values of $\mu_{1}$ are in the suitability region of $s_{j}^{2}$, whereas for $\mu_{1}$ close to 1 , the series approximations are not suitable. The suitability region grows with the order of the partial sum, as shown in Figures 17 and 18. 


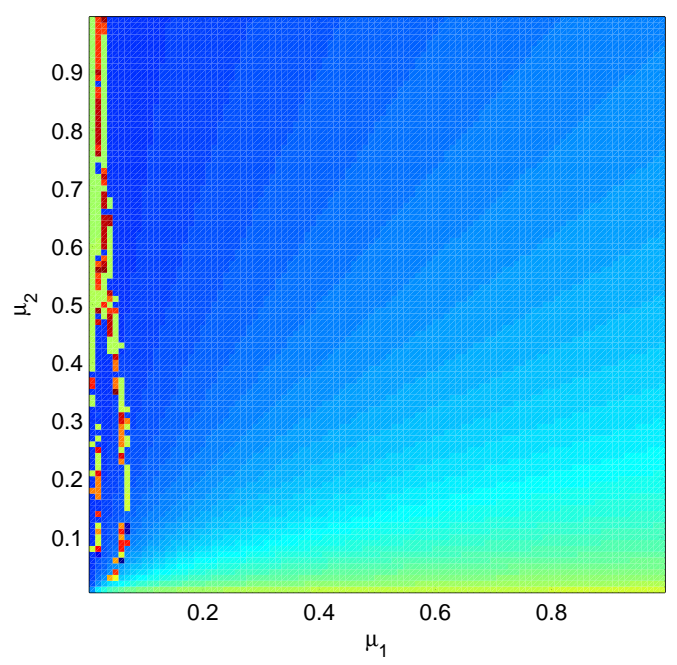

Figure 13: Suitability region for $S_{1}$ of the first degree approximation $s_{1}^{1}$.

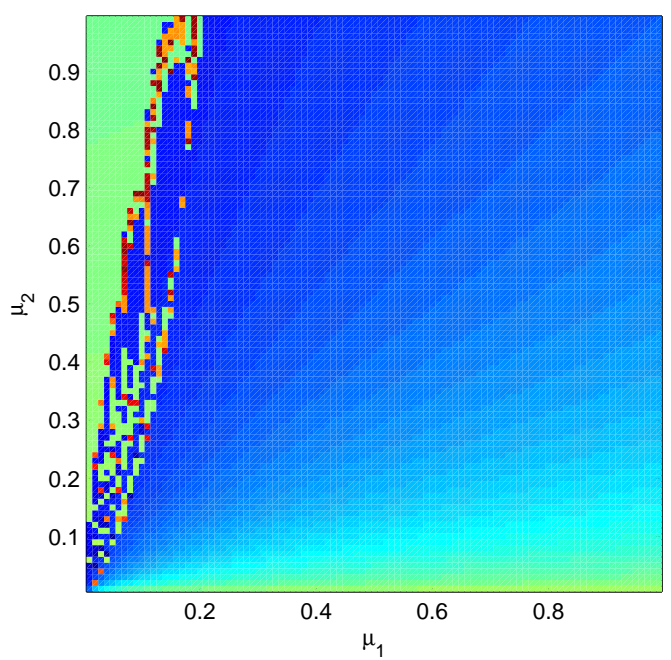

Figure 14: Suitability region for $S_{1}$ of the third degree approximation $s_{3}^{1}$. 


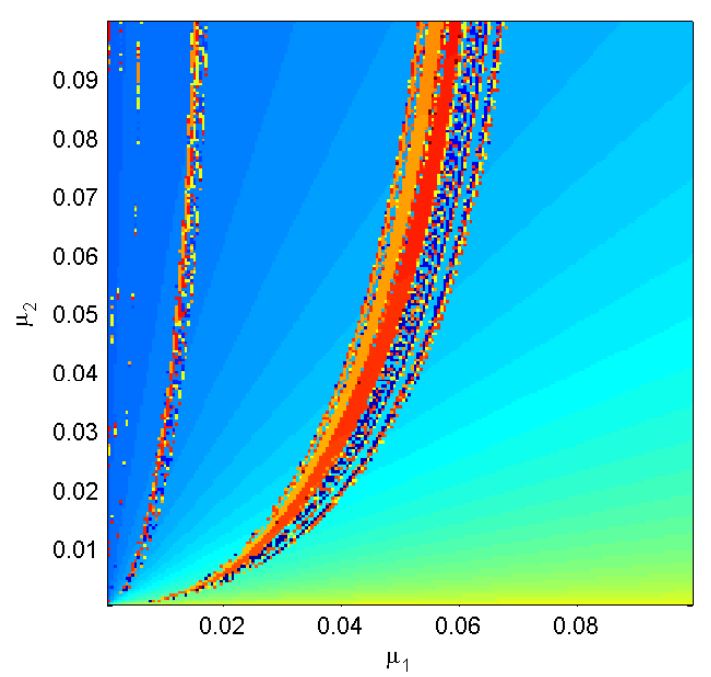

Figure 15: Detail of the $S_{1}$-suitability region of $s_{1}^{1}$ near the origin.

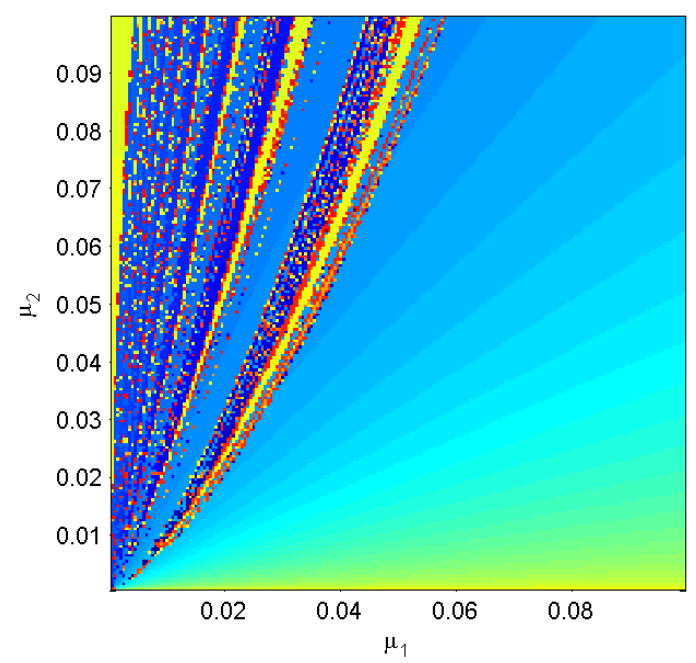

Figure 16: Detail of the $S_{1}$-suitability region of $s_{3}^{1}$ near the origin. 


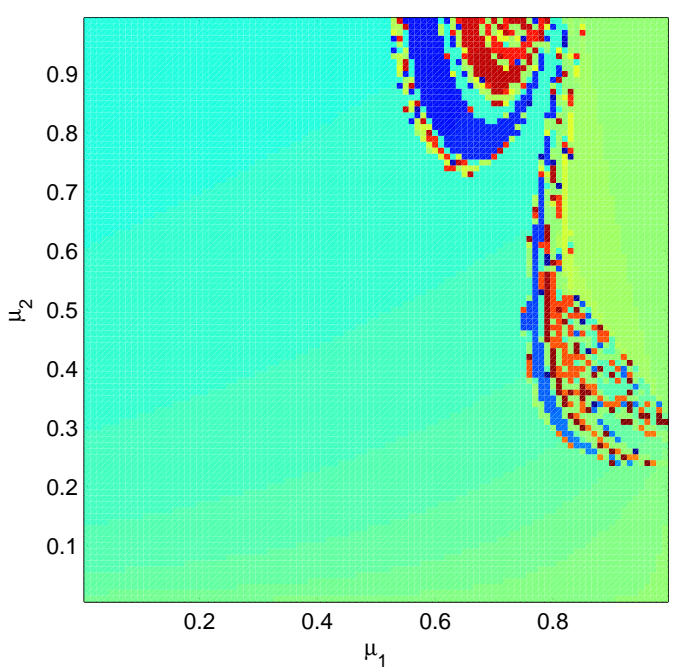

Figure 17: Suitability region for $S_{2}$ of the approximation $s_{1}^{2}$.

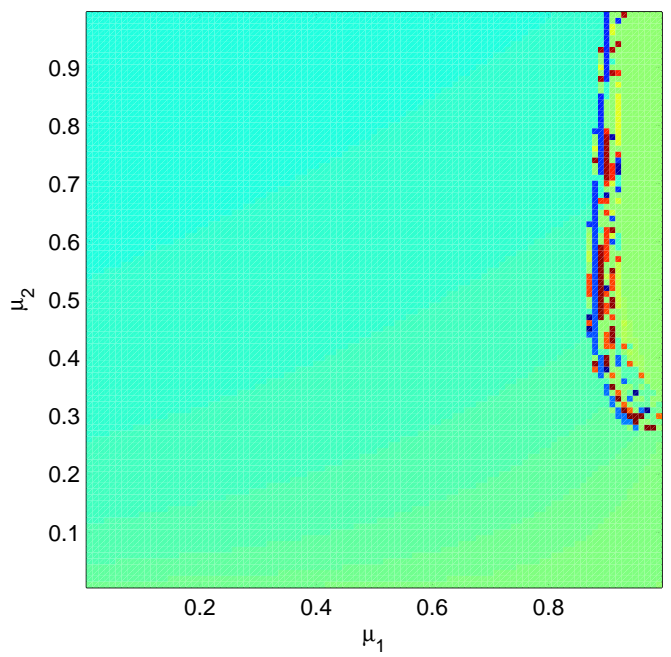

Figure 18: Suitability region for $S_{2}$ of the approximation $s_{3}^{2}$. 


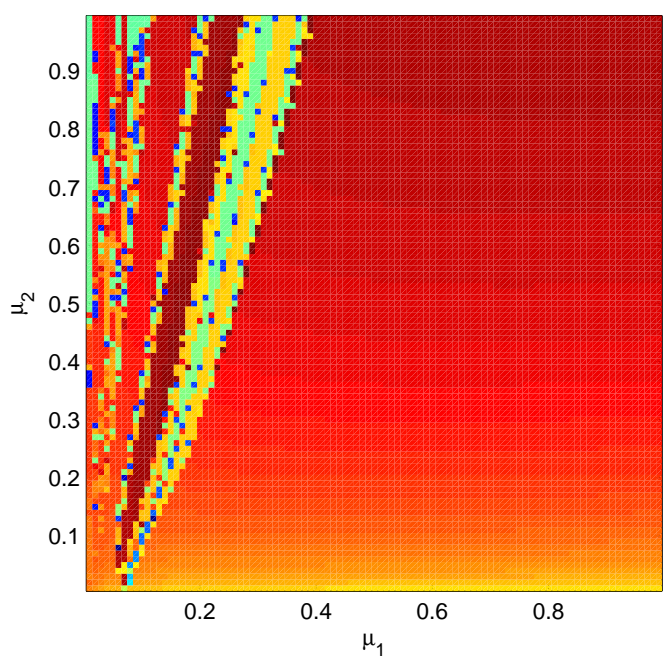

Figure 19: Suitability region for $S_{4}$ of the approximation $s_{1}^{4}$.

The behaviour for $S_{4}$ is similar to that of $S_{1}$, but in this case, the suitability region shrinks as the order increases (see Figures 19 and 20).

The series (10) produces in general $S_{7}$-suitable estimates, but they are not better than the zero term of the series, $(1 / 2,-\sqrt{3} / 2)$, which is $S_{7}$ suitable for almost all $\left(\mu_{1}, \mu_{2}\right)$. There is only a small region where the convergence to $S_{7}$ is not guaranteed for the zero term, for $0<\mu_{1}<0.01$, whereas taking as starting points higher order terms of the series $s_{j}^{-}$, the convergence requires conditions such as $\mu_{2}<\mu_{1}$. Figures 21 and 22 show the suitability regions for the zero term, and for the fifth order term, respectively. In the first one, the unsuitability zone is difficult to appreciate, whereas the second figure presents two zones of convergence to different solutions and an intermediate zone comprised between two lines, where the behaviour of the iterations is more complex. In Figure 23 you can see a magnification of the non-suitability region for the zero term that appears for very small values of $\mu_{1}$ but reaches relatively high values of $\mu_{2}$.

\section{Conclusions}

We have analysed the attraction basins of the equilibrium solutions of the restricted four-body problem (1). Due to the complexity of the attraction basins, it is difficult to guarantee that a starting point will converge to the desired solution. By using series expansions one can obtain approximations for the solutions that can be used as starting point for Newton's iterations. We have presented a graphical analysis of the behaviour of different series approximations obtaining the suitability regions for different orders and equilibrium points. In particular, we have seen that the partial sums of series (10) do not provide better estimations for Newton's method than its zero term. Summing up, 


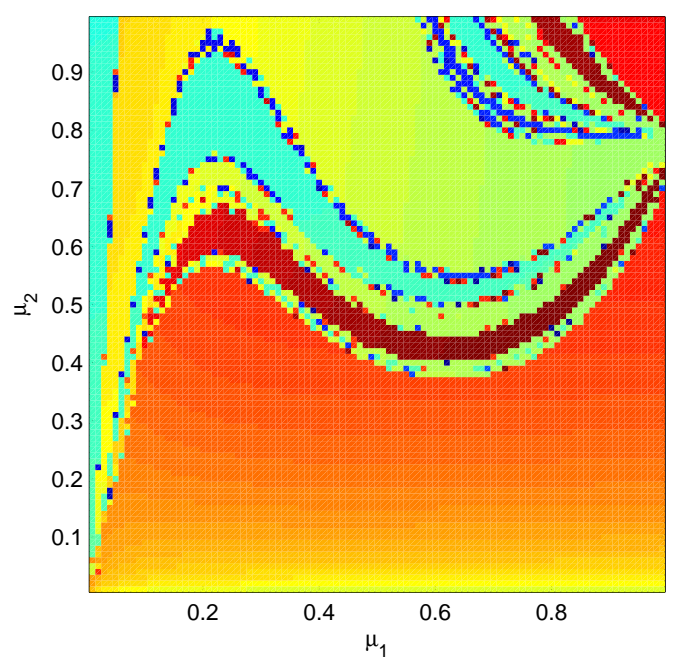

Figure 20: Suitability region for $S_{4}$ of the approximation $s_{3}^{4}$.

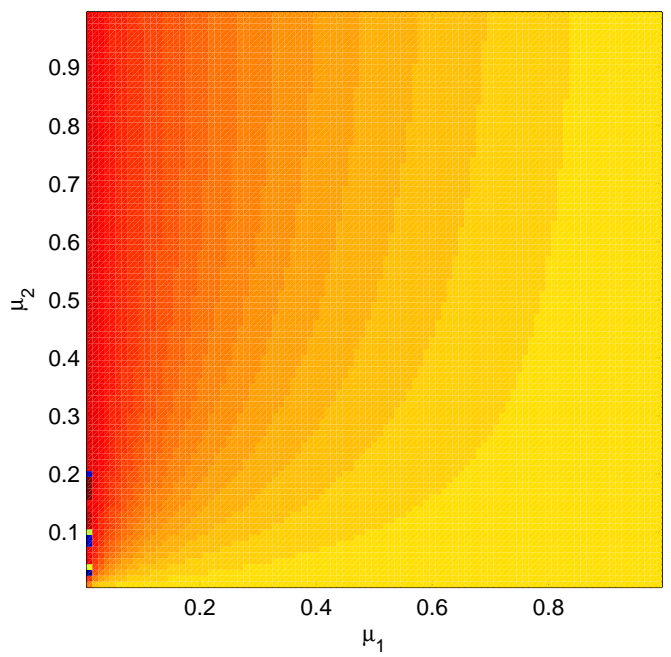

Figure 21: Suitability region for $S_{7}$ of the approximation $s_{0}^{-}$. 


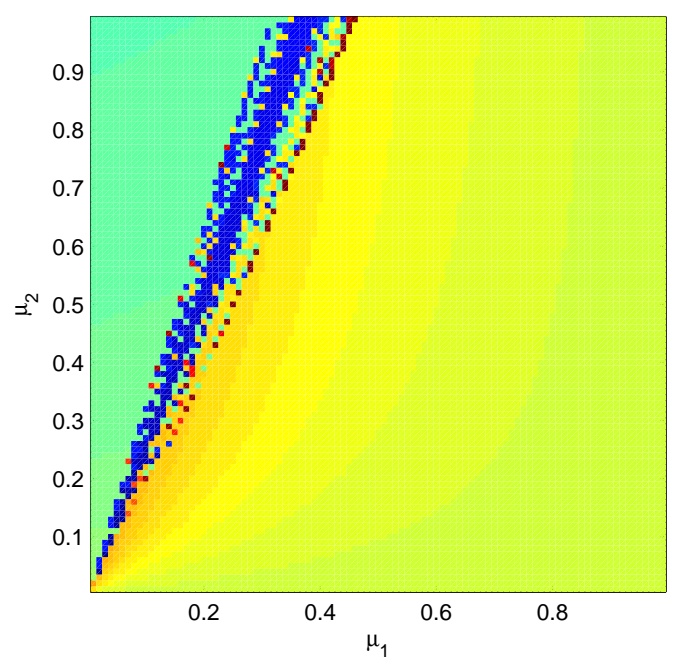

Figure 22: Suitability region for $S_{7}$ of the approximation $s_{5}^{-}$.

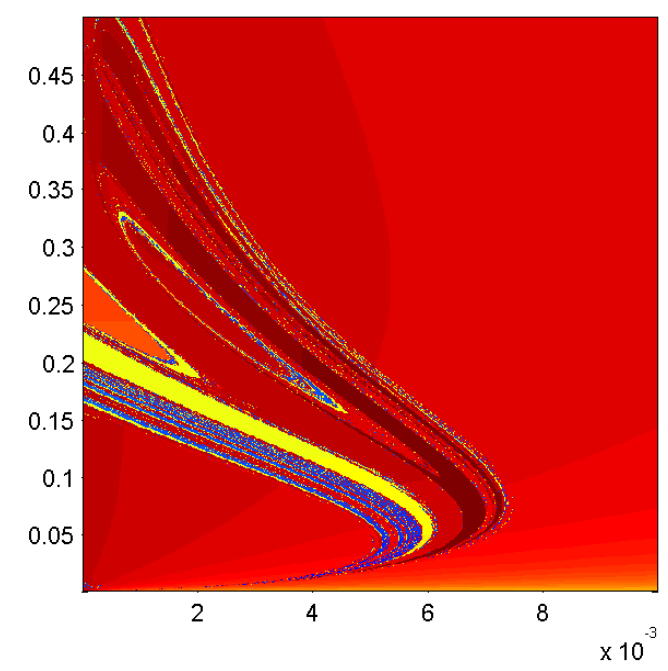

Figure 23: Unsuitability zone for $S_{7}$ of the zero term $s_{0}^{-}$. 
we have studied the non-trivial situation when applying Newton's method for finding numerical solutions of the nonlinear system that describes a real application in celestial mechanics. A priori, it is difficult to guarantee the convergence to the required solution.

\section{References}

[1] J.L. Varona, Graphic and numerical comparison between iterative methods, Math. Intelligencer, 24, pp. 37-46 (2002).

[2] S. Amat, S. Busquier, S. Plaza, Chaotic dynamics of a third-order Newton-type method, Journal of Mathematical Analysis and Applications 366-1, pp. 24-32 (2010).

[3] S. Amat, S. Busquier, Á.A. Magreñán, Reducing Chaos and Bifurcations in Newton-Type Methods, Abstract and Applied Analysis 2013, 10 pp., dx.doi.org/10.1155/2013/726701

[4] E. Bedford, J. Diller, Real and complex dynamics of a family of birational maps of the plane: the golden mean subshift. Amer. J. Math. 127(2005), pp. 595-646.

[5] R. Durán, J. Muñoz, A. Peinado, Classifying quadratic maps from plane to plane, Linear Algebra Appl., 364 (2003), pp. 1-12.

[6] P. Montel, Leçons sur les familles normales, Gauthier-Villars, (1927).

[7] D.A. Budzko, A.N. Prokopenya, Symbolic-Numerical Methods for Searching Equilibrium States in a Restricted Four-Body Problem. Programming and Computer Software, Vol. 39:2, pp. 74-80 (2013).

[8] C. Simó, Relative equilibrium solutions in the four body problem. Celestial Mechanics, 18, pp. 165-184 (1978).

[9] F. Diacu, The solution of the n-body problem. Mathematical Intelligencer. 18,(3), pp. 66-70 (1996).

[10] J.F. Traub, Iterative methods for the solution of equations, Chelsea Publishing Company, New York, (1982).

[11] D.A. Budzko, A.N. Prokopenya, Symbolic-numerical analysis of equilibrium solutions in a restricted four-body problem. Programming and Computer Software, Vol. 36:2, pp. 68-74 (2010).

[12] G. B. Thomas, R.L. Finney, Calculus and Analytic Geometry, 9th ed., AddisonWesley, (1996).

[13] K.F. Sundman, Memoire sur le probleme des trois corps. Acta mathematica, 36.1, pp. 105-179 (1913).

[14] V. Szebehely, Theory of orbits: the restricted problem of three bodies. Academic Press, New York, (1967). 